\title{
Análisis de minimización de costos de infusiones endovenosas aplicadas en pacientes hospitalizados y pacientes ambulatorios en el servicio de reumatología en un hospital de Lima, Perú
}

\author{
Cost minimization analysis of intravenous infusions applied in inpatient and outpatient \\ rheumatology service at a hospital in Lima, Peru \\ Clara Gallegos-Vergara ${ }^{1,2}$, Pablo Best-Bandenay ${ }^{2}$, Michelle Lozada-Urbano ${ }^{3,4}$
}

\section{RESUMEN}

Objetivo: Medir los costos de la terapia de infusión endovenosa hospitalaria vs ambulatoria en el servicio de Reumatología, del Hospital Nacional Edgardo Rebagilati Martins (HNERM) de Lima Perú, en el año 2009. Métodos: El diseño corresponde a un estudio de tipo descriptivo, retrospectivo y transversal. La población estuvo comprendida por todos los pacientes hospitalizados $(N=42)$, que recibieron infusiones endovenosas con rituximab, pamidronato, infliximab, abatacept, tocilizumab, ácido zoledrónico y ciclofosfamida en el servicio de Reumatología. Los gastos médicos y no médicos directos corresponden a un año. Se elaboró una ficha, en la que se solicitó información de tipo socioeconómica y demográfica. Se identificaron los costos marginales de las alternativas. Resultados: La población se caracterizó por ser en mayor proporción de sexo femenino, con nivel de instrucción superior universitaria, y con predominancia de las enfermedades reumáticas en el grupo etáreo de 42 a 52 años en adelante. El costo total anual de la hospitalización ascendió a S/.472 726,21 (US\$ 166 160,30 ); y para la alternativa ambulatoria de S/.416 092,25 (US\$146 253,867), los cuales hacen una diferencia de S/.56 633,96 (US\$ 19.906,49) que equivale al 12\%. La administración de las infusiones endovenosas ambulatoriamente (en asegurados que no tienen criterios de hospitalización), permitió disminuir los costos suscitados por la hospitalización (hotelería, alimentación y personal). La mayor diferencia en éste estudio lo establecieron los costos de hotelería (día cama), costo de personal y costo de pérdida de productividad. Conclusiones: La administración de infusiones endovenosas ambulatoriamente tuvo un menor costo para la institución y para el asegurado.

Palabras clave: Evaluación económica, costo mínimo, costo, infusiones endovenosas, reumatología.

\section{ABSTRACT}

Objective: To measure the costs of therapy hospital intravenous infusion. vs outpatient service of Rheumatology, the National Hospital Edgardo Rebagilati Martins (HNERM) in Lima Peru, in 2009. Methods: The study design was a descriptive, retrospective and transversal. The population was comprised of all hospitalized patients $(\mathrm{N}=42)$ who received intravenous infusions of rituximab, pamidronate, infliximab, abatacept, tocilizumab, zoledronic acid and cyclophosphamide in the service of Rheumatology. Inclusion criteria were to be of both sexes over thirteen policyholders and / or beneficiaries of EsSalud. Medical and non-medical direct costs are for one year. A chip was developed, in which socioeconomic and demographic information requested type. The marginal costs of the alternatives were identified. Results: The study population was characterized by a greater proportion of female university level higher education, and prevalence of rheumatic diseases in the age group of 42-52 years and up. The total annual cost of hospitalization amounted to S / 472 726,21 (US \$ 166 160,355); and for outpatient alternative to S / 416 092,25 (US \$ 146 253,867), which make a difference in S / 56633,96 (US \$ 19 906,49) is equivalent to $12 \%$ savings. The management of outpatient intravenous infusions (in policyholders who have no hospitalization criteria) allowed reduce costs arising from hospitalization (hotel, food and staff). The biggest difference in this study was established hospitality costs (day bed), personnel costs and cost of lost productivity. Conclusions: The administration of intravenous infusions outpatients had lower costs for the institution and for the insured, thus optimizing resources.

Keywords: Economic evaluation, cost minimization analysis, cost, intravenous infusions, rheumatology.

\footnotetext{
${ }^{1}$ Hospital Nacional Edgardo Rebagliati Martins

${ }^{2}$ Universidad Peruana Cayetano Heredia. Lima, Perú

${ }^{3}$ Universidad Peruana de Ciencias Aplicadas. Lima, Perú

${ }^{4}$ Universidad Nacional José Faustino Sánchez Carrión. Huacho, Perú
} 


\section{INTRODUCCIÓN}

Enfermedad inflamatoria sistémica, la artritis reumatoide (AR) es de curso crónico, de causa multifactorial y origen autoinmune, cuya mayor caracterización es dolor y daño articular con progresiva discapacidad. Se estima que en el mundo la prevalencia de enfermedades reumáticas alcanza el $0,5-1,0 \%$ en la población adulta (Kvien, 2004). La prevalencia en Perú alcanza el $0,5 \%$ y se manifiesta predominantemente en mujeres en un intervalo que varía entre los 45 a 65 años (AcevedoVásquez, 2012; Gamboa et al., 2007).

En la actualidad, una alternativa de grandes expectativas por su efectividad en pacientes con AR es la terapia con biológicos (Fan y Leong, 2007). Las estimaciones de los costos médicos directos son sustancialmente mayores que las estimaciones de costos antes de la era de terapia biológica, y los costos están impulsadas principalmente por el costo de los medicamentos, agentes principalmente biológicos (Michaud, Messer, Choi, y Wolfe 2003). El incremento de los costos se da por la introducción y mayor uso de biofosfonatos y quimioterápicos (Becerra, et al., 2011), (Rituximab, Infliximab, Tocilizumab, Abatacept Pamidronato, Ácido zoledrónico y Ciclofosfamida).

En Argentina los costos totales atribuibles a la Espondilitis Anquilosante alcanzan los \$6,720 (pesos argentinos) anuales. Los costos indirectos representaron alrededor del $70 \%$, siendo la discapacidad funcional el principal factor asociado a mayor costo (Marengo y Schneeberger, 2009).

Un estudio de análisis de minimización de costos (AMC) de quimioterapia oral e intravenosa en pacientes con cáncer de pulmón, nueva formulación oral de VNB. La efectividad clínica es determinada por costos de adquisición de medicamentos, seguimiento, administración, gestión de toxicidad y transporte del paciente, correspondiente al tratamiento intravenoso. Mientras que la administración de VNB oral, permite la autoadministración en el hogar cada 21 días, ahorrando costos de recursos hospitalarios (Le Lay, Myon, Hill, y Riou-Franca, 2007).
En el Perú existe una evaluación costo efectividad donde el costo del tratamiento con Abatacept es de S/. 169263 (Becerra, et al., 2011). Estudios en el Servicio de Reumatología del HNERM- EsSalud, indican que las cinco primeras causas de hospitalización del año 2009 fueron: Artritis Reumatoide (29\%), Lupus Eritematoso Sistémico (27\%), Espondilitis Anquilosante (11\%), Esclerosis sistémica y Vasculitis (10\%) (Chigne, 2004).

Los tiempos usados para colocar la infusión endovenosa en pacientes con AR con infliximab, fueron estudiados en dos momentos (G1 tratamiento en 2 horas, y G2 infliximab de 30 a 45 minutos), ha demostrado que en ambos grupos no ocurrió variación en las funciones vitales, demostrando que la aplicación de la terapia rápida de 30 a 45 minutos no representa riesgos para la salud del paciente. La presencia de efectos secundarios no fue significativa en relación con la velocidad de infusión (EsSalud HNERM 2009).

Administraron Infliximab a los pacientes con AR de manera ambulatoria, esto resultó en una mejoría clínica significativa a las $48 \mathrm{~h}$. Los pacientes que toleraron las infusiones de infliximab en más de $2 \mathrm{~h}$ también toleran la infusión en $1 \mathrm{~h}$ (Shergy, et al., 2002).

Este estudio plantea determinar cuál de las alternativas para la aplicación de las infusiones endovenosas es en términos de costo menor, la administración durante la hospitalización o la administración de manera ambulatoria de los pacientes del servicio de reumatología atendidos en el año 2009.

\section{MATERIAL Y MÉTODOS}

El diseño corresponde a un estudio de tipo descriptivo, retrospectivo y transversal. La población estuvo comprendida por todos los pacientes hospitalizados en el servicio de Reumatología ( $N=42$ ), que recibieron infusiones endovenosas en el año 2013. Como criterios de inclusión debían ser de ambos sexos, mayores de trece años asegurados y/o derechohabientes de EsSalud. Los criterios de exclusión, fueron pacientes menores de trece años y los que han recibido algún de tipo de medicamento por patología no reumática.

La información fue tomada directamente de los registros e historias clínicas de los pacientes que acudieron al servicio de hospitalización de 
Reumatología del HNERM con problemas reumáticos y que ingresaron única y exclusivamente para tratamiento con la terapia mencionada (usando precios del 2009).

\section{Infusiones endovenosas aplicadas en pacientes hospitalizados y en pacientes ambulatorios}

En el servicio de reumatología del HNERM, los tratamientos farmacológicos son suministrados por vía endovenosa a través de un catéter periférico de manera continua o intermitente por un período de tiempo. Los pacientes hospitalizados son aquellos que acuden a su terapia y permanecen en el hospital 24 horas. El modelo de tratamiento a los pacientes ambulatorios, incluye todo el proceso y solo se quita el costo por hospitalización.

\section{Análisis de minimización de costos (AMC)}

EI AMC involucra la determinación de la cantidad de dinero requerido para entregar un servicio sin relación con el resultado del paciente, así pues para desarrollar el AMC, un requisito previo es el establecimiento de la similitud (igual efectividad) de los resultados de las opciones tratamiento que se cumple en este estudio. El objetivo de este tipo de análisis está en encontrar la manera menos costosa de alcanzar un resultado. Cuando los costos o los beneficios no son constantes por unidad de producción para alguna de las alternativas evaluadas, se debe realizar un análisis marginal de costes y beneficios.

\section{Recolección de datos}

\section{Paciente ambulatorio}

La información del paciente ambulatorio $(n=42)$ se hizo con un modelo hipotético de la actividad que podría desarrollarse si se implementara este proceso. Los costos directos del paciente, consulta, gastos por transporte y gasto farmacéutico. Los costos indirectos corresponde a los días gastados por la espera de cama, pérdida de producción por los días que estuvo hospitalizado y los días que permaneció convaleciente en casa, los datos correspondientes al salario fueron imputados. Los costos intangibles son aquellos que se calculan con criterios subjetivos y que no son registrados como costos en los sistemas (Rodríguez, 2000).

\section{Cálculo de costos para EsSalud}

Se calculó costo día cama, costos de medicamentos, materiales (según tipo de patología).

\section{Cálculo de costos de infusiones endovenosas}

Se realizó el cálculo de costos de infusiones endovenosas, teniendo en cuenta la frecuencia de suministro de la terapia y número de días de hospitalización.

\section{Costo de los recursos humanos}

Para determinar el cálculo del costo de remuneración de los recursos humanos (médico, enfermera y técnico de enfermería) que intervienen en el proceso de hospitalización y en el proceso ambulatorio (hipotético), se consideró la variación según niveles remunerativos, cuyas escalas están diferenciadas en cinco niveles para los diferentes grupos ocupacionales, tomándose como promedio el nivel 3 , se realizó los cálculos del costo minuto que emplea el recurso humano, para proporcionar atención en cada intervención según niveles ocupacionales.

La depreciación de la moneda no fue significativa, por lo tanto no se considera la inflación en el análisis. Tampoco se ha considerado la depreciación del mobiliario porque tienen más de 30 años de antigüedad por lo tanto, las existentes están depreciadas.

\section{Cálculo de costos para el acompañante}

Dentro de los costos directos para el acompañante se encuentra el costo por transporte, se efectuó un estimado en relación a la frecuencia que el acompañante estuvo con el paciente buscando cama para su hospitalización, así como también durante la estancia hospitalaria del paciente, para efectuar el cálculo de estos costos se tomó como referencia la remuneración mínima vital. Dentro de los costos indirectos para el acompañante encontramos la pérdida de productividad, para realizar el cálculo se toma en cuenta los días y horas que estuvo acompañado al paciente antes y durante la hospitalización en razón a la remuneración mínima vital. 


\section{Cálculo de minimización de costos}

Con los datos obtenidos de costos, se realizó el análisis de las siguientes alternativas: hospitalizado (alternativa 1 ) versus ambulatorio (alternativa 2).

Según el siguiente cálculo:

$(\mathrm{Cd} 1+\mathrm{Cl} 1)-(\mathrm{CD} 2+\mathrm{Ci} 2)$

Donde:

CD1 = costos directos de la alternativa 1 (hospitalizado)

CD2 $=$ costos directos de la alternativa 2 (ambulatorio)

$\mathrm{Cl} 1=$ costos indirectos de la alternativa 1 (hospitalizado)

$\mathrm{Cl} 2=$ costos indirectos de la alternativa 2 (ambulatorio)

\section{Cálculo de Costo Marginal}

El costo marginal (CMg), que se define como el costo adicional de producir una unidad más de producto o servicio.

Se realizó el siguiente cálculo:

$\mathrm{CMg}=\mathrm{CT}(\mathrm{Q}+1)-\mathrm{CT}(\mathrm{Q})$

Dónde:

$\mathrm{CT}=$ costo total

$\mathrm{Q}=$ coste de producir una cantidad determinada de producto o de servicio.

Este estudio fue aprobado por el Comité Institucional de Ética de la Universidad Peruana Cayetano Heredia.

\section{Efectividad clínica de las intervenciones, evidencia bibliográfica}

En relación a las referencias bibliográficas que están evidenciando que las intervenciones tienen efectividades clínicas similares, en la publicación Effectiveness of biologic therapies for rheumatoid arthritis: an indirect comparisons approach, se estudia y compara la eficacia de los fármacos biológicos antirreumáticos, (DMARDs) versus placebo, con y sin metrotexato. Este estudio sugiere que los DMARD biológicos son igualmente eficaces, los resultados mencionan que el punto final para medir eficacia fue usar el criterio de mejora del $50 \%$ según el Colegio Americano de Reumatología. (Devine, Alfonso-Cristancho y Sullivan, 2011).

Bañuelos (2007), hace mención que la ausencia de efectos secundarios destacables asociados a la reducción del tiempo de infusión de infliximab, permite señalar que la infusión rápida puede ser un método a tener en cuenta para optimizar las prestaciones de los hospitales de estancia corta para terapias biológicas. Razón por la cual consideramos viable el desarrollo del estudio minimización de costos de las modalidades ambulatorio y hospitalario, donde se identificará la alternativa de menor costo o más eficiente en la administración de infusiones endovenosas.

En el suministro del tratamiento tanto ambulatorio como hospitalario; la dosificación, la vía de administración y el medicamento, no varían de acuerdo a lo prescrito por los profesionales del área, es decir, no se modifica lo indicado médicamente, solamente la modalidad del suministro (ambulatorio u hospitalario), considerándose efectividades clínicas equivalentes (Ceteris Paribus), tal como mostramos en la tabla siguiente: 
Tabla 1. Descripción del suministro de los tratamientos y estudios que respaldan la efectividad clínica.

\begin{tabular}{|c|c|c|c|c|c|c|}
\hline \multirow{2}{*}{$\begin{array}{l}\text { Paciente y } \\
\text { patología }\end{array}$} & \multirow{2}{*}{ Medicamento } & \multirow{2}{*}{ Técnica } & \multicolumn{2}{|c|}{ Tratamiento } & \multirow{2}{*}{ Comentario } & \multirow{2}{*}{ Bibliografía } \\
\hline & & & Hospitalario & Ambulatorio & & \\
\hline \multirow{20}{*}{ A. Reumatoide } & \multirow{5}{*}{ Abatacept } & $\begin{array}{l}\text { Vía de } \\
\text { administración }\end{array}$ & Endovenoso & Endovenoso & El mismo & \multirow{5}{*}{$\begin{array}{l}\text { Maya, H. } \\
\text { (2008) } \\
\text { Genovese, } \\
\text { M.C. (2008) } \\
\text { Russell, A.S } \\
\text { (2007) }\end{array}$} \\
\hline & & Dosis & Idéntica & Idéntica & $\begin{array}{l}\text { Cálculo de acuerdo } \\
\text { al peso del paciente }\end{array}$ & \\
\hline & & $\begin{array}{l}\text { Tiempo de } \\
\text { entrega de servicio }\end{array}$ & $>$ Tiempo & $<$ Tiempo & Difiere & \\
\hline & & $\begin{array}{l}\text { Estancia hospitalaria } \\
\text { (costo día cama) }\end{array}$ & Sí & No & $>$ Costo hospitalario & \\
\hline & & $\begin{array}{l}\text { Pérdida de productividad } \\
\text { del paciente }\end{array}$ & Sí & No & $\begin{array}{l}\text { Tiempo perdido de las } \\
\text { actividades productivas } \\
\text { PEA }\end{array}$ & \\
\hline & \multirow{5}{*}{ Tocilizumab } & $\begin{array}{l}\text { Vía de } \\
\text { administración }\end{array}$ & Endovenoso & Endovenoso & El mismo & \multirow{5}{*}{$\begin{array}{l}\text { Maini, R.N. } \\
(2006)\end{array}$} \\
\hline & & Dosis & Idéntica & Idéntica & $\begin{array}{l}\text { Cálculo de acuerdo } \\
\text { al peso del paciente }\end{array}$ & \\
\hline & & $\begin{array}{l}\text { Tiempo de } \\
\text { entrega de servicio }\end{array}$ & >Tiempo & $<$ Tiempo & Difiere & \\
\hline & & $\begin{array}{l}\text { Estancia hospitalaria } \\
\text { (costo día cama) }\end{array}$ & Sí & No & $>$ Costo hospitalario & \\
\hline & & $\begin{array}{l}\text { Pérdida de productividad } \\
\text { del paciente }\end{array}$ & Sí & No & $\begin{array}{l}\text { Tiempo perdido de las } \\
\text { actividades productivas } \\
\text { PEA }\end{array}$ & \\
\hline & \multirow{5}{*}{ Rituximab } & $\begin{array}{l}\text { Vía de } \\
\text { administración }\end{array}$ & Endovenoso & Endovenoso & El mismo & \multirow{5}{*}{$\begin{array}{l}\text { Ribby, W. } \\
(2010)\end{array}$} \\
\hline & & Dosis & Idéntica & Idéntica & $\begin{array}{l}\text { Cálculo de acuerdo } \\
\text { al peso del paciente }\end{array}$ & \\
\hline & & $\begin{array}{l}\text { Tiempo de } \\
\text { entrega de servicio }\end{array}$ & >Tiempo & $<$ Tiempo & Difiere & \\
\hline & & $\begin{array}{l}\text { Estancia hospitalaria } \\
\text { (costo día cama) }\end{array}$ & Sí & No & $>$ Costo hospitalario & \\
\hline & & $\begin{array}{l}\text { Pérdida de productividad } \\
\text { del paciente }\end{array}$ & Sí & No & $\begin{array}{l}\text { Tiempo perdido de las } \\
\text { actividades productivas } \\
\text { PEA }\end{array}$ & \\
\hline & \multirow{5}{*}{ Infliximab } & $\begin{array}{l}\text { Vía de } \\
\text { administración }\end{array}$ & Endovenoso & Endovenoso & El mismo & \multirow{5}{*}{$\begin{array}{l}\text { Kiortsis, D.N. } \\
(2006) \\
\text { Schiff, D.N. } \\
(2008)\end{array}$} \\
\hline & & Dosis & Idéntica & Idéntica & $\begin{array}{l}\text { Cálculo de acuerdo } \\
\text { al peso del paciente }\end{array}$ & \\
\hline & & $\begin{array}{l}\text { Tiempo de } \\
\text { entrega de servicio }\end{array}$ & >Tiempo & $<$ Tiempo & Difiere & \\
\hline & & $\begin{array}{l}\text { Estancia hospitalaria } \\
\text { (costo día cama) }\end{array}$ & Sí & No & $>$ Costo hospitalario & \\
\hline & & $\begin{array}{l}\text { Pérdida de productividad } \\
\text { del paciente }\end{array}$ & Sí & No & $\begin{array}{l}\text { Tiempo perdido de las } \\
\text { actividades productivas } \\
\text { PEA }\end{array}$ & \\
\hline \multirow{5}{*}{$\begin{array}{l}\text { B. Lupus } \\
\text { eritematoso } \\
\text { sistémico }\end{array}$} & \multirow{5}{*}{ Ciclosfosfamida } & $\begin{array}{l}\text { Vía de } \\
\text { administración }\end{array}$ & Endovenoso & Endovenoso & El mismo & \multirow{5}{*}{$\begin{array}{l}\text { Ramos, M. } \\
(2005)\end{array}$} \\
\hline & & Dosis & Idéntica & Idéntica & $\begin{array}{l}\text { Cálculo de acuerdo } \\
\text { al peso del paciente }\end{array}$ & \\
\hline & & $\begin{array}{l}\text { Tiempo de } \\
\text { entrega de servicio }\end{array}$ & $>$ Tiempo & $<$ Tiempo & Difiere & \\
\hline & & $\begin{array}{l}\text { Estancia hospitalaria } \\
\text { (costo día cama) }\end{array}$ & Sí & No & $>$ Costo hospitalario & \\
\hline & & $\begin{array}{l}\text { Pérdida de productividad } \\
\text { del paciente }\end{array}$ & Sí & No & $\begin{array}{l}\text { Tiempo perdido de las } \\
\text { actividades productivas } \\
\text { PEA }\end{array}$ & \\
\hline
\end{tabular}




\begin{tabular}{|c|c|c|c|c|c|c|}
\hline \multirow{10}{*}{ C. Osteoporosis } & \multirow{5}{*}{ Pamidonato } & $\begin{array}{l}\text { Vía de } \\
\text { administración }\end{array}$ & Endovenoso & Endovenoso & El mismo & \multirow{10}{*}{ Jin, M. (2010) } \\
\hline & & Dosis & Idéntica & Idéntica & $\begin{array}{l}\text { Cálculo de acuerdo } \\
\text { al peso del paciente }\end{array}$ & \\
\hline & & $\begin{array}{l}\text { Tiempo de } \\
\text { entrega de servicio }\end{array}$ & $>$ Tiempo & $<$ Tiempo & Difiere & \\
\hline & & $\begin{array}{l}\text { Estancia hospitalaria } \\
\text { (costo día cama) }\end{array}$ & Sí & No & $>$ Costo hospitalario & \\
\hline & & $\begin{array}{l}\text { Pérdida de productividad } \\
\text { del paciente }\end{array}$ & Sí & No & $\begin{array}{l}\text { Tiempo perdido de las } \\
\text { actividades productivas } \\
\text { PEA }\end{array}$ & \\
\hline & \multirow{5}{*}{$\begin{array}{l}\text { Ácido } \\
\text { Zolendrónico }\end{array}$} & $\begin{array}{l}\text { Vía de } \\
\text { administración }\end{array}$ & Endovenoso & Endovenoso & El mismo & \\
\hline & & Dosis & Idéntica & Idéntica & $\begin{array}{l}\text { Cálculo de acuerdo } \\
\text { al peso del paciente }\end{array}$ & \\
\hline & & \multirow{3}{*}{$\begin{array}{l}\text { Tiempo de } \\
\text { entrega de servicio } \\
\text { Estancia hospitalaria } \\
\text { (costo día cama) } \\
\text { Pérdida de productividad } \\
\text { del paciente }\end{array}$} & $>$ Tiempo & $<$ Tiempo & Difiere & \\
\hline & & & Sí & No & $>$ Costo hospitalario & \\
\hline & & & Sí & No & $\begin{array}{l}\text { Tiempo perdido de las } \\
\text { actividades productivas } \\
\text { PEA }\end{array}$ & \\
\hline
\end{tabular}

\section{RESULTADOS}

El mayor número de pacientes recibieron infliximab y rituximab en su tratamiento por AR, espondilitis anquilosante (EA) y lupus eritematoso sistémico (LES). La población en estudio estuvo comprendida por 42 pacientes hospitalizados, en el servicio de Reumatología del HNERM en el año 2009, para recibir infusión endovenosa con ciclofosfamida, rituximab, abatacept, infliximab, ácido zoledrónico, tocilizumab y pamidronato

Tabla 2. Características socioeconómicas y demográficas de la población, 2009

\begin{tabular}{lrc}
\hline Variables & N & $\%$ \\
\hline Población total & & \\
Atendidos & 42 & 11,9 \\
Atenciones & 82 & 19 \\
Grupo edad & 5 & 286 \\
$20-30$ & 8 & 26,2 \\
$31-40$ & 12 & 14,3 \\
$42-52$ & 11 & 47,6 \\
$53-63$ & 6 & 52,4 \\
$64-74$ & 20 & 18,6 \\
Sexo & 22 & 9,3 \\
Masculino & & 60,5 \\
Femenino & 8 & 11,6 \\
Grado de instrucción & 4 & 26 \\
Secundaria completa & 5 & \\
Superior técnica & & \\
Superior universitaria & & \\
Postgrado & & \\
\hline
\end{tabular}

Fuente: Archivo de Historias clínicas HNERM 2009

En la Tabla 2 se observa que la población es mayormente de sexo femenino (52,4\%), también resalta la frecuencia de las enfermedades reumáticas en el grupo etario de 42 a 52 años $(28,6 \%)$ con grado de instrucción a predominancia superior universitaria (62\%). 
Tabla 3. Tipo de costos y determinación de costos marginales según tipo de infusión endovenosa: Hospitalización vs Ambulatorio, 2009.

\begin{tabular}{|c|c|c|c|c|c|c|c|c|c|}
\hline & \multicolumn{3}{|c|}{ EsSalud } & \multicolumn{2}{|c|}{ Paciente } & \multicolumn{2}{|c|}{ Acompañante } & \multirow{3}{*}{$\begin{array}{l}\text { Costo total } \\
\text { por infusión } \\
\text { (nuevos } \\
\text { soles) }\end{array}$} & \multirow{3}{*}{$\begin{array}{c}\text { Diferencia } \\
(\%)\end{array}$} \\
\hline & \multicolumn{3}{|c|}{ Costos Directos } & \multirow{2}{*}{$\begin{array}{c}\text { Costos } \\
\text { Indirecto } \\
\text { Pérdida de } \\
\text { productividad }\end{array}$} & \multirow{2}{*}{$\begin{array}{l}\text { Costos } \\
\text { Directo } \\
\text { Costo de } \\
\text { transporte }\end{array}$} & \multirow{2}{*}{$\begin{array}{c}\text { Costos } \\
\text { Indirecto } \\
\text { Pérdida de } \\
\text { productividad }\end{array}$} & \multirow{2}{*}{$\begin{array}{l}\text { Costos } \\
\text { Directo } \\
\text { Costo de } \\
\text { transporte }\end{array}$} & & \\
\hline & Día cama & $\begin{array}{l}\text { Medicinas y } \\
\text { materiales }\end{array}$ & Personal & & & & & & \\
\hline \multicolumn{10}{|l|}{ Pamidronato } \\
\hline Hospitalización & 117,39 & 113,9 & 318,8 & 289,63 & 122,1 & 229,2 & 252,3 & 1443,32 & \\
\hline Ambulatorio & 0 & 110,72 & 198 & 110,7 & 98,4 & 24,7 & 98,4 & 640,97 & $55,60 \%$ \\
\hline Costo marginal & 117,39 & 3,18 & 120,8 & 178,93 & 23,7 & 204,45 & 154,9 & 802,35 & \\
\hline \multicolumn{10}{|l|}{ Rituximab } \\
\hline Hospitalización & 234,78 & 8448,06 & 461,65 & 264,94 & 45,3 & 220 & 29,6 & 9704,33 & \\
\hline Ambulatorio & 0 & 8440,72 & 132,144 & 42,8 & 36,5 & 15 & 19,4 & 8686,564 & $10,40 \%$ \\
\hline Costo marginal & 234,78 & 7,34 & 329,506 & 222,14 & 8,8 & 205 & 10,2 & 1017,766 & \\
\hline \multicolumn{10}{|l|}{ Ciclofosfamida } \\
\hline Hospitalización & 234,78 & 63,33 & 307,9 & 95,86 & 10,6 & 256,7 & 31,5 & 1000,7 & \\
\hline Ambulatorio & 0 & 58,89 & 66,072 & 6,62 & 5,33 & 9,2 & 5,3 & 151,412 & $84,90 \%$ \\
\hline Costo marginal & 234,78 & 4,44 & 241,8 & 89,24 & 5,27 & 247,5 & 26,2 & 849,3 & \\
\hline \multicolumn{10}{|l|}{ Abatacep } \\
\hline Hospitalización & 234,78 & 3381,66 & 256,75 & 110 & 36,4 & 330 & 984 & 4447,99 & \\
\hline Ambulatorio & 0 & 3380,54 & 16,5 & 3,6 & 16,8 & 3 & 168 & 3437,24 & $22,70 \%$ \\
\hline Costo marginal & 234,78 & 1,12 & 240,25 & 106,4 & 19,6 & 327 & 816 & 1010,75 & \\
\hline \multicolumn{10}{|l|}{ Infliximab } \\
\hline Hospitalización & 117,39 & 7936,1 & 216,2 & 299,7 & 70 & 369,3 & 125,1 & 9133,79 & \\
\hline Ambulatorio & 0 & 7935,72 & 66,072 & 36 & 28,5 & 5,5 & 28,5 & 8100,292 & $11,30 \%$ \\
\hline Costo marginal & 117,39 & 0,38 & 150,128 & 263,7 & 41,5 & 363,8 & 96,6 & 1033,498 & \\
\hline \multicolumn{10}{|l|}{$\begin{array}{l}\text { Ácido } \\
\text { Zolendrónico }\end{array}$} \\
\hline Hospitalización & 117,39 & 85,86 & 130,65 & 117,48 & 11,2 & 82,5 & 10 & 555,08 & \\
\hline Ambulatorio & 0 & 85,27 & 16,5 & 4,75 & 9,6 & 1,5 & 6 & 123,62 & $77,77 \%$ \\
\hline Costo marginal & 117,39 & 0,59 & 114,15 & 112,73 & 1,6 & 81 & 4 & 431,46 & \\
\hline
\end{tabular}

En la Tabla 3 se muestra los costes totales por cada tipo de infusión, para EsSalud, el paciente y acompañante, según hospitalización y ambulatoriamente, estableciéndose un estándar por cada tratamiento. El costo del personal de salud y la pérdida de productividad del paciente correspondió al tiempo de suministro del tratamiento pudiendo ser de 30min a $6 \mathrm{~h}$ de acuerdo al tipo de infusión endovenosa.

Para efectuar la valoración económica, se realizó un análisis de costos según el tipo de infusión endovenosa (ciclofosfamida, pamidronato, ácido zoledrónico, abatacept, rituximab, infliximab) suministrado en el Servicio de Reumatología.

La diferencia en los costos marginales es altamente significativa tal como ocurre con el pamidronato siendo $\mathrm{S} / .802,35$ nuevos soles que equivale al $55,6 \%$ entre la alternativa hospitalaria y ambulatoria. También podemos citar a la ciclofosfamida siendo su costo marginal de $\mathrm{S} / .849,3$ nuevos soles que equivale al $84,9 \%$. 
Tabla 4. Determinación del Costo Total Anual en Hospitalización vs. Ambulatorio

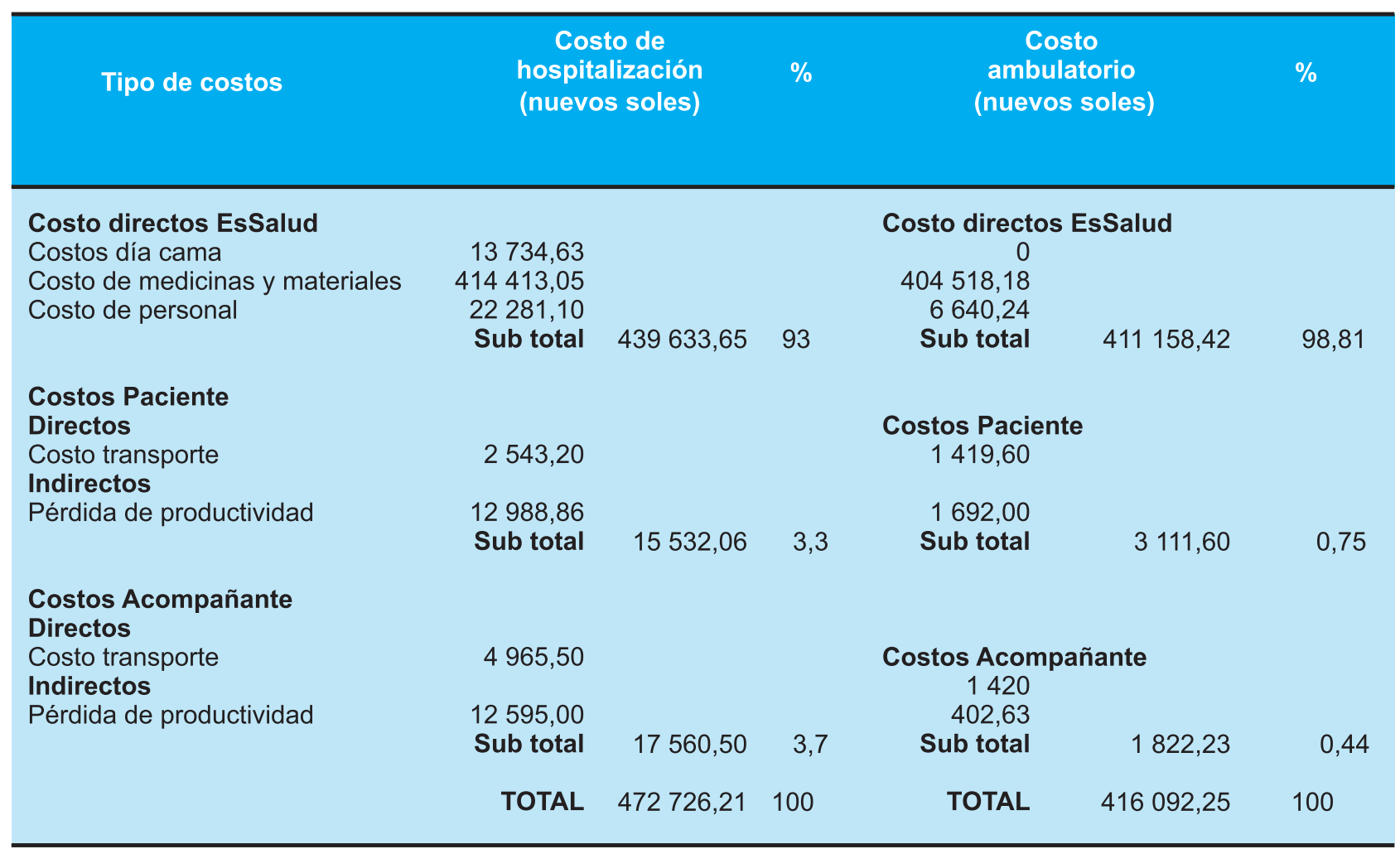

Los costos anuales totales se muestran en la Tabla 4, para cada alternativa, el costo total anual en hospitalización es mayor el cual asciende a S/.472 726,21; en cambio el costo total anual para la alternativa ambulatoria fue menor ascendiendo a S/.416 092,25. Haciendo una diferencia de $S / .56$ 633,96 que equivale al $12 \%$ de ahorro. La mayor diferencia en este estudio lo establecen el costo de hotelería (día cama), costo de personal y costo de pérdida de productividad.

\section{DISCUSIÓN}

Este estudio obtuvo un ahorro económico en la administración de infusiones endovenosas ambulatorias en comparación con las hospitalarias, equivalente al $12 \%$ anual, demostrando que si hubiéramos atendido a este mismo grupo de pacientes ambulatoriamente la institución hubiese ahorrado S/.56 633,96 nuevos soles.

El costo directo total anual de la infusión endovenosa en hospitalización se incrementó debido a los costos de hotelería (día cama) representado por el $3 \%$ del costo global y costos de personal equivalente al $5 \%$ del total. Mientras si el mismo grupo de pacientes hubiese recibido su tratamiento ambulatoriamente el costo por pérdida de productividad ascendería al $0,4 \%$ del total, y gastos por transponte $0,3 \%$ del costo global. Asimismo el gasto del acompañante por pérdida de productividad y transporte ascendieron a $0,1 \%$ y $0,3 \%$ respectivamente.

La mayoría de estudios de costos encontrados, enfocan los aspectos del prestador del servicio de salud, dejando de lado la perspectiva del paciente que no determina los gastos directos e indirectos que estos realizan para su tratamiento.

Dentro de los costos indirectos y directos encontramos que el asegurado y acompañante durante la hospitalización dejaron de asistir a su centro laboral, ocasionando pérdida de productividad representado por $3 \%$ del costo global, en ambos casos. Los gastos por transporte del paciente y acompañante ascendieron al $1 \%$ del total.

Se obtuvo los costos marginales para cada infusión endovenosa sujeta de estudio, denotando el incremento de los costes al producir un determinado servicio en la administración de ciclofosfamida, rituximab, 
abatacept, ácido zoledrónico, pamidronato e infliximab en hospitalización.

La aplicación de la alternativa ambulatoria permitirá lograr un uso racional de los recursos de la institución, disminuyendo así costos innecesarios generados por los gastos de hospitalización, esto permitirá una mayor disponibilidad de las instalaciones y camas para los usuarios que reúnan los criterios de hospitalización para el servicio; y los recursos humanos para la realización de otras actividades asistenciales, generando una utilización adecuada de los recursos disponibles, basada en las necesidades del servicio y la demanda de los usuarios.

La población de pacientes que reciben infusiones endovenosas en el servicio de Reumatología estuvo conformada por personas aseguradas del régimen contributivo de EsSalud; que en general tienen un grado de instrucción a predominio superior universitaria; lo que permitiría una mayor aceptación de los beneficios personales de la aplicación ambulatoria; como el evitar la pérdida de días productivos y laborales, menor exposición a infecciones intrahospitalarias (Parada, 2001) y menor tiempo de permanencia hospitalaria.

En cuanto al ahorro del tiempo la investigación permite establecer ventajas en la aplicación de los procedimientos en el área asistencial, permitiendo abarcar una mayor atención a la demanda asegurada hospitalizada (PacíficoBedón, 2007). Asimismo, el seguimiento y tratamiento ambulatorio de la enfermedad es la mejor inversión pues garantiza mejores resultados terapéuticos, mejor calidad de vida y un ahorro monetario sustancial para el paciente; siendo los medicamentos empleados los que representan una proporción importante del costo total (Montoya y Gómez, 2011).

El cálculo de los costos totales de la hospitalización y para la alternativa ambulatoria ascendió a S/.472 726.21 (US\$ 166 160.355); de S/.416 092.25 (US\$ $146 \quad 253.867$ ) respectivamente, los cuales hacen una diferencia de S/.56,633.96 (US\$ 19 906.49) que equivale al $12 \%$ de ahorro.

En nuestro estudio los costos por paciente hospitalizados alcanzó la suma en dólares de 3 956,0 y por año 166160.36 (\$ USD de 2013), siendo superiores a los encontrados en Canadá, donde los costos de pacientes con AR van desde 2 299,0 por pacientes con AR por año a 13549 (\$ USD de 1996), el estudio se centra solo en los pacientes que han sido hospitalizados de igual manera en nuestro estudio (Lubeck, 2001).

\section{REFERENCIAS BIBLIOGRÁFICAS}

Acevedo-Vásquez, EM. (2012). Algunos aspectos de la artritis reumatoide en Perú. Rev Soc Peru Med Interna, 25 (1), 31-37.

Bañuelos, D. (2007). Infliximab en infusión Rápida. Eficacia y complicaciones. Reumatología Clinica, 3(4).

Becerra, F., Benites, C., Aiello, E., Zingoni, C., Bergman, G., Drost, P. \& Sanabria, C. (2011). Costo efectividad de Abatacept en comparación con otras terapias biológicas para el tratamiento de la artritis reumatoide moderada a severamente activa en pacientes que han fallado al tratamiento con metotrexato en EsSalud para el año 2010. Acta méd. peruana [online]. 28 (4), citado desde 2014-0713], pp. 200-210. Disponible en: $<$ http://www.scielo.org.pe/scielo.php?scr ipt $=\mathrm{sci}$ arttext\&pid=S 1728 $59172011000400005 \&$ lng=es\&nrm=iso> ISSN 1728-5917.

Chigne, O. (2004). Costos en pacientes con artritis reumatoide en monoterapia con metotrexate según respuesta terapéutica y calidad de vida. Rev. Perú. reumatol, 10 (3), 6-18.

Devine, E., Alfonso-Cristancho, R. \& Sullivan, S. (2011). Effectiveness of biologic therapies for rheumatoid arthritis: an indirect comparisons approach. Pharmacotherapy: The Journal of Human Pharmacology and Drug Therapy, 31, (1), 39-51.

ESSALUD - HNERM. (2009). Oficina de Estadística-RAR.

Fan, P.T. \& Leong, K. H. (2007). The use of biological agents in the treatment of rheumatoid arthritis. Ann Acad Med Singapore, 36,128-34. 
Le Lay, K., Myon, E., Hill, S. \& Riou-Franca, L. (2007). Comparative cost-minimisation of oral and intravenous chemotherapy for first-line treatment of non-small cell lung cancer in the UK NHS system. Eur $J$ Health Econ, 8, 145-151.

Lubeck, D.P. (2001). A review of the direct costs of rheumatoid arthritis: managed care versus fee-for-service settings. Pharmacoeconomics, vol 19 (8), 811-8.

Marengo, M. \& Schneeberger, E. (2009). Impacto socioeconómico en pacientes con espondilitis anquilosante en Argentina. Revista reumatológica de Argentina, Año 20 (3), 20-25

Michaud, K., Messer, J., Choi, H.K. \& Wolfe, F. (2003). Direct medical costs and their predictor in patients with rheumatoid arthritis. Arthritis Rheum, 48, 2750-2762.

Montoya, N. \& Gómez, L. (2011). Costos directos del tratamiento de pacientes con artritis reumatoide en Medellín, Colombia. Revista colombiana de Reumatología, 18 (1), 26-33.

Shergy, W.J., Isern, R.A., Cooley, D.A., Harshbarger, J.L., Huffstutter, J.E., Hughes, G.M et al. (2002). Open label study to assess infliximab safety and timing of onset of clinical benefit among patients with rheumatoid arthritis. J Rheumatol, 29 (4), 667-77.

Parada, I. (2001). Aspectos a considerar en el análisis de los costos de la atención médica. Hitos de Ciencias Económico Administrativas, 19, 41-46.

Pacífico-Bedón, J. (2007). Estudio de utilización de medicamentos biológicos en el Servicio de Reumatología del Hospital Nacional Edgardo Rebagliati Martins. Departamento Académico de Farmacología, Bromatología y Toxicología-UNMSM.

Rodríguez, E. (2000). Costos en salud: del análisis contable a la evaluación económica. Revistas de Ciencias Administrativas y Financieras de la Seguridad Social, $8(1)$.

\section{Correo electrónico:}

claragallegosv@hotmail.com

Revisado por pares:

Recibido: 05-04-2015

Aprobado: 24-06-2015 